\title{
Domingo Faustino Sarmiento y Francisco Miró-Quesada Cantuarias: Vanguardistas del pensamiento político y el filosofar latinoamericano \\ Domingo Faustino Sarmiento and Francisco Miró-Quesada Cantuarias: Avant-gardes of political thought and Latin American philosophy
}

\author{
Leonardo Díaz-Pertuz \\ Idiazpertuz@gmail.com \\ Universidad del Sinú Elías Bechara Zainum, Córdoba \\ Colombia \\ https://orcid.org/0000-0002-8588-1075 \\ Luz Estela Díaz-Cid \\ ledic64@hotmail.com \\ Universidad del Sinú Elías Bechara Zainum, Córdoba \\ Colombia \\ https://orcid.org/0000-0002-1500-3031 \\ Luz Marina-Mejía \\ andreucci luz@hotmail.com \\ Universidad Dr. Rafael Belloso Chacín, Zulia \\ Venezuela \\ https://orcid.org/0000-0002-1546-6730 \\ Miguel Negrón \\ miguelnegronf@gmail.com \\ Universidad Dr. Rafael Belloso Chacín, Zulia \\ Venezuela \\ https://orcid.org/0000-0003-3054-0457
}

Recibido: 01 de abril de 2020

Aprobado: 20 de julio de 2020 


\title{
RESUMEN
}

La presente investigación tuvo como propósito fundamental destacar la influencia de Domino Faustino Sarmiento y Francisco Miró-Quesada Cantuarias, como vanguardistas del pensamiento político y el filosofar latinoamericano. Se enmarca dentro del enfoque post-positivista; en el paradigma cualitativo-interpretativo y con un diseño bibliográfico, asumiendo el método hermenéutico para el análisis de los contenidos teóricos del material estudiado. Como conclusión, se puede expresar que, aunque ambos autores desempeñaron su labor en siglos y épocas distintas; se consideran vanguardistas en los ámbitos de la educación, la política la filosofía y la educación universitaria desde sus ideas y proposiciones enmarcadas en la naturaleza del hombre como base para el progreso y desarrollo de los pueblos.

Palabra Claves: Educación; filosofía cultural; desarrollo cultural; pensamiento crítico.

\begin{abstract}
ABSTRAC
The main purpose of this research was to highlight the influence of Domino Faustino Sarmiento and Francisco Miró-Quesada Cantuarias, as Vanguardists of Political Thought and Latin American philosophizing. It is framed within the post-positivist approach; in the qualitative-interpretive paradigm and with a bibliographic design, assuming the hermeneutical method for the analysis of the theoretical contents of the material studied. As a result, it was found that both authors carried out their work in different centuries and times. In conclusion, they can be considered avant-garde in the fields of education, politics, philosophy and university education based on the nature of man as the basis for the progress and development of peoples.
\end{abstract}

Key Words: Education; cultural philosophy, cultural development; critical thinking.

\section{INTRODUCCIÓN}

Domingo Faustino Sarmiento, nació en el siglo XIX en Argentina y cubrió con sus huellas gran parte de este tiempo desde los escenarios de la docencia, la literatura y el periodismo. Asimismo fue político, militar y estadista, desempeñándose primero como gobernador de San Juan desde 1862 hasta 1864 y más tarde ocupando la presidencia de Argentina por un periodo de 6 años a partir de 1864 .

Asimismo, este autodidacta es reconocido en su país natal como el maestro de escuela que se convirtió en presidente; y dado que desde muy joven participara políticamente en oposición al Dictador Juan Manuel Rosas. Sin embargo, su gran 
pasión fue la educación llegando a ser Rector de la Escuela Normal de Preceptores de Chile. Trascendió tanto su obra en Latinoamérica en el campo de la educación y la política que el Congreso Panamericano de Ministros de Instrucción Pública, reunidos en Panamá en 1943, aprobó el 11 de septiembre, fecha de su desaparición física, como el Día del Maestro de América en reconocimiento de la grandeza de este Maestro en la vida intelectual de Hispanoamérica.

Por motivos políticos, emigró a Chile con toda la familia incluyendo a sus padres. Allí se distinguió por constituir escuelas, periódicos, espacio de encuentros literarios y de análisis político, siempre con su estilo confrontativo con el régimen de su país, razón por la cual ganó muchas críticas y antipatías de sus adversarios. Con la ayuda de políticos amigos, entre ellos Manuel Monnt Torres es designado comisionado por el Gobierno de Chile para estudiar la educación y los métodos pedagógicos en Europa y Norteamérica, logrando una densa formación intelectual en las áreas humanísticas. Como político en función de gobierno, Sarmiento, demostró ser un vanguardista al introducir grandes cambios en la educación y en administración pública en general de Argentina. Además de ser un promotor de la inmigración controlada, estimulando la participación de ciudadanos de otras latitudes en importantes proyectos de creación de instituciones que traerían progreso a su país.

Es digno destacar, que ha mediado del siglo XIX estuvo en pleno apogeo la revolución industrial, época en la cual termino la etapa feudal, caracterizada por la verticalidad; es así como se establecen las relaciones con el gran número de personas o grupos muy variados, como corresponde a quien ejercer el Poder. En este contexto, el presidente Sarmiento, fue prolífico en su obra, creó importantes instituciones de carácter científico y promovió la creación de dos significativos medios de comunicación de masas. Entre sus obras públicas más importantes están: la construcción de los ferrocarriles con una red ferroviaria de $1.300 \mathrm{Km}$; la construcción de puentes y vías de penetración agrícolas; el desarrollo de la navegabilidad de ríos; y, como realización trascendental, al finalizar su administración inauguró el cable transoceánico. 
Para Ocampos, con relación a Domingo Sarmiento, es necesario destacar que:

Sus coterráneos recuerdan su obra como la de un gran civilizador autoritario, centralizador del poder ejecutivo y con gran decisión para culturizar a Argentina; fue el gran difusor de la enseñanza primaria y un gran promotor de la inmigración, fiel a su campaña de «poblar el desierto». Sus numerosas obras, artículos de revistas y periódicos, discursos e informes fueron recopilados en 53 volúmenes, que señalan su profunda y variada producción literaria, que lo llevan a ser considerado como uno de los grandes pensadores de Hispanoamérica en el siglo XIX" (Ocampos, 2000: p. 9).

Por otra parte, en la primera mitad del siglo XX, América Latina se distingue por la gran influencia de la filosofía desde Aristóteles hasta Hegel. Pero, es de saber que históricamente, fue Miro Quesada quien se sitúa en la realidad del continente, para romper con esta dependencia intelectual exponiendo los trabajos de brasileros intelectuales renombrados; filósofo y matemático con un valor agregado al destacar la importancia filosófica en la tecnológica. En la actualidad estos trabajos siguen siendo fundamentos de los sistemas informáticos, pudiéramos decir que Mirò Quesada fue vanguardista en la nueva manera de filosofar, no solo para este continente, sino para el mundo.

Como intelectual de envergadura y con visión de futuro, Miró Quesada se preocupó por introducir desde el Perú y para el Sub-Continente, la lógica y las corrientes epistemológicas contemporáneas, enfrentado así el neo-coloniaje; expresando que en el siglo que le tocó vivir "la filosofía es la ciencia que ha fracasado, y la ciencia es la filosofía que ha triunfado". Como izquierdista cromosómico, pensaba que no existía línea divisoria entre la Unión soviética y China, y entre otras naciones, dentro de la órbita del comunismo Euro-asiático.

Miró-Quesada, se destaca por su forma de pensar la filosofía, especialmente en el campo de la lógica matemática, la relación entre la matemática y la filosofía y algunos problemas científico-filosóficos. Abrazó el comunismo, influenciado por su estadía en países que comulgaban con esta ideología. Se preocupó por estudiar el pensamiento filosófico latinoamericano de manera autentica; generándole la necesidad de crear un proyecto de filosofía que forjara un despertar ante las corrientes del pensamiento con pretensiones de hegemonizar esta parte del mundo. 
Al respecto, Quiroz (2007) expresa que Miró-Quesada, su terco humanismo y su búsqueda de una fundamentación racional de la ética, trasunta su ideología humanista, que en términos concretos lo había llevado antes a ser el ideólogo del partido gobernante en el Perú, Acción Popular, en los años 60; atravesando varias etapas de su pensamiento, pero hilado por una constante vocación democrática y educativa ( $p$ 259)

Esta breve semblanza demuestra que con Francisco Miró Quesada Cantuarias, Latinoamérica cuenta con un pensador que se identifica con el suelo que pisa, pero que entiende que el problema de la autenticidad de la filosofía no es solamente concentrarse en una realidad del contexto, sino que se debe abocar a una dimensión mayor; el problema del hombre en su integralidad, lo que lo hace más complejo y universal.

Considerando lo planteado, se presenta el siguiente articulo con la intención de mostrar la influencia de Domino Faustino Sarmiento y Francisco Miró-Quesada Cantuarias, como pensadores vanguardistas en el ámbito de la política y la filosofía Latinoamericana. Para lograr este cometido se realizó una investigación bibliográfica amparada en el paradigma cualitativo-interpretativo, con un diseño bibliográfico. Por consiguiente, se asumió el método hermenéutico para el análisis de los contenidos teóricos en relación al material bibliográfico de estudio seleccionado.

\section{DESARROLLO}

\section{La América Latina del siglos: XIX y XX}

Desde el ámbito de las ciencias y la filosofía, América Latina se ha debatido entre llegar a la modernización sin perder la identidad; es más se ha esmerado en el reforzamiento de la misma, quizás marcado por los siglos antecesores de dominación europea y norteamericana. En este sentido, nos atrevemos a expresar que la segunda se ha impuesto ante la primera. Hoy se observa un descenso en la onda modernizadora y un ascenso en la perspectiva identitaria. Vale decir, la globalización con su desbastadora influencia en los patrones culturales de cada región del mundo 
donde ha logrado permear a todo evento ha conseguido, en esta parte del sur del globo terráqueo, una barrera.

Desde este sentir histórico, Villarán (2019) expresa que "Miró Quesada es uno de los filósofos más importantes de la historia del Perú-sin duda, de los más productivos y originales" (p. 1340). Sus aportes se basan en aspectos políticos como: la democracia y la inmigración, el espiritualismo, la lucha contra el utilitarismo, su exaltación de las humanidades y la latinidad. A este le siguen pensadores con diferentes posturas, pero con un denominador común, la pugna por mejoras en lo ético-social. Esta fue conocida como una inclinación anarquista, que llegó a transformarse en un tema de significativa relevancia en los círculos de discusión, en América Latina, llegando a tener eco en los gobiernos de turno; representada, entre otros, por José Ingenieros, quien planteó que los intelectuales deben participar en la emancipación de todos los ciudadanos.

Según, Cumana (2008, p. 835) la propuesta de Ingenieros por una sociedad más equitativa se puede apreciar en lo siguiente" la explicación de la desigualdad social, moral y racial e inclusive, justifica la discriminación por edad al incluir a los niños entre los "salvajes y primitivos", En este sentido, la cuestión social

Asimismo, se puede afirmar que Mariátegui, fue el pensador de esta época más universal de los marxistas latinoamericanos, enmarca sus reflexiones en el ámbito cultural para tratar de entender la formación social peruana de entonces, modelando cambios sociales posibles, con influencia indígena. Además, su inclinación por el marxismo italiano le permite valorar la complejidad de la cultura. Por lo que el propio Mariátegui, expone "no soy un crítico imparcial y objetivo, mis juicios se nutren de mis ideales, de mis sentimientos, de mis pasiones" (Peña, 2008, p. 40).

Miró Quesada, en la actualidad alinea su pensamiento con quienes considera que la intelectualidad debe estar al servicio de las grandes causas sociales, pero desde la propia realidad del contexto local, libre de dominaciones enajenantes. Está consciente que la vía más eficaz para la liberación del hombre latinoamericano es a través del dominio de la racionalidad y desalinear, siendo la condición más permanente de recuperar la plena humanidad mediante la anuencia de la razón. Esta perspectiva, que destaca un periodo importante de su vida, lo conllevó a ver la filosofía de la liberación 
como la manera de ir a la búsqueda de la filosofía estrictamente original de América Latina. Sin embargo, la propia esencia histórico-universal de la filosofía hizo que se abandonase tal proyecto.

\section{La Educación como eje del desarrollo social}

Cuando se compara el nivel de desarrollo que haya alcanzado cualquier país del planeta y se relaciona con la inversión en educación e investigación se logra determinar una correspondencia directa en ambas variables. De hecho los Organismos multilaterales, en sus informes anuales, registran cifras e índices que demuestran reiterativamente esta reciprocidad. Así, en el informe de seguimiento de la educación en el mundo UNESCO (2016, p. 42), expresa: "sistema educativo ocupa un lugar central para el desarrollo social inclusivo; la educación puede dotar a las personas de los conocimientos, competencias y valores necesarios para mejorar los resultados en el ámbito social y cambiar las normas de la sociedad".

Esta planteamiento actual, extrapolado al contexto epocal de Domingo Sarmiento, muestra lo consciente que estaba para el momento de la importancia de la educación para la humanidad y lo indispensable de un sistema educativo sólido para logar una sociedad de hombres libres e iguales, y que supieran lo bastante como para tener conciencia de su dignidad y de su derecho a la libertad. Ya Hobbes, en el siglo XVII, había planteado que el hombre en sus estado natural y en su libre albedrio sintió la necesidad de crear normas de convivencias que elevara los niveles de socialización y alejara a los individuos del salvajismo. Esta etapa pre jurídica primitiva dio inicio a la estructura del Estado.

Al respecto, Arias, interpretando el pensar de Sarmiento, explana:

Esta disposición de su pensamiento a partir de dos ejes: uno positivo y otro negativo, que se oponen, le permite un cuestionamiento de la realidad social y el planteo de un proyecto transformador en el que triunfa la civilización. Ello requiere otorgar a la educación el papel dinamizador de la sociedad y exigir al Estado la directa intervención en ese ámbito, ya que, desde su perspectiva, la escuela pública sistematizaría la destrucción de la barbarie y estructuraría una nueva sociedad. (Arias, 2011: p 14) 
Por su parte, Miro-Quesada, comprometido con su sociedad; sobre todo, por haber desempeñado diversos cargos internacionales y prestados importantes servicios a la organización pública, tales como ministro de Educación, aportando especialmente a la filosofía, en área de las matemáticas, a la jurídica y al formalismo ético; asimismo fue un defensor de la filosofía latinoamericana y estudioso de la naturaleza el hombre; atendiendo primero el conocimiento y luego los asuntos ético y político.

\section{El pensamiento universal de Domingo Faustino Sarmiento.}

La dimensión continental de Sarmiento fue apuntalada desde la política, como autodidacta y acérrimo opositor junto a su familia a la dictadura de Rosas. Ya exilado en Chile se dedicó con más fuerza a la docencia, al periodismo y a la política, por supuesto. Esto último le facilito una misión por el encaro del Gobierno de Chile, que le permitió madurar intelectualmente, gracias a sus viajes por Europa y Estados Unidos de Norteamérica.

A Pesar de sus éxitos, Sarmiento asume la filosofía del pesimismo; reflexiona sobre las razas americanas, otorgándole a la colonización española la categoría de una tara genética sobre los pueblos al sur de la América, diferenciándola de la población del norte continental, a quienes considera con mayor capacidad por su diferencia en cuanto a los colonizadores. En este estado, pregunta: ¿Y cuál es el camino para cambiar la sangre y la mentalidad del pueblo hispanoamericano? La inmigración y la educación.

Según Sarmiento (1995), con inmigrantes: provenientes del viejo continente de Europeo: alemanes, ingleses, españoles italianos, franceses, entre otros; la raza hispanoamericana se fortalecerá en la ciencia y la cultura enrumbándose hacia el progreso.

El europeo trae consigo una parte de la ciencia, de la industria y de los medios mecánicos de producir de las naciones civilizadas; de donde resulta que cuantos más europeos acudan a un país, más se irá pareciendo ese país a la Europa hasta que llegue un día en que le sea superior su riqueza con población y en industria, cosa que ya sucede hoy en los Estados Unidos. 
Por otra parte, Sarmiento puntualiza que la educación debe ser la primera empresa del Estado; pues sostiene que es necesario educar a los pueblos, no solamente a las élites o grupos superiores, sino a las grandes mayorías o masas populares. Con la educación, no solamente se aprende la cultura de los pueblos, los valores y las tecnologías, sino que se concreta la auténtica independencia y libertad de los pueblos hispanoamericanos.

\section{El pensamiento universal del Miró Quesada}

La obra de Francisco Miró Quesada (1974), se basa fundamentalmente en tratar de explicar ontológicamente si en realidad existe una filosofía que se pueda considerar propia de América Latina. Para ello debe salvar el primer gran escollo: la filosofía es comprensión, intangibilidad e historia, por lo tanto sólo puede hacerse desde una perspectiva histórica que trascienda de generación a generación, contando siempre con un antecedente. De lo anterior entonces, surge la necesidad de estas interrogantes: ¿Existen en América Latina reflexiones filosóficas trascendentales precursoras? ¿La situación del continente en vías de desarrollo ha marcado las reflexiones filosóficas a través del tiempo?

Lo expresado, es considerado como un reto a su pensamiento angustiado por la motivación de comprender las corrientes europeas del momento. Se ve obligado a pensar sobre artificios y realidades extrañas; proponiéndose, en primer término, el abordaje de las tendencias del momento histórico, el análisis de las variables que le permitieran entender al Occidente y más tarde la recuperación de la antigüedad, de las condiciones mediatas y últimas de esta comprensión.

Tales exigencias llegan a ser tan agudas en el latinoamericano, que empieza incluso a aplicárselas a su propia realidad. Para recuperar la propia tradición filosófica comienza a considerar libros cuyos contenidos recoges las posturas de diferentes autores sobre la historia del continente americano en el marco de un horizonte prometedor.

De todos estos retos, con el norte firme de la autenticidad de nuestra gente, germina la idea de diseñar una estrategia que le permitiera a un grupo de intelectuales latinoamericanos expresar en sus obras el pensamiento filosófico propio de estos 
escenarios. Este movimiento recuperativo, abre caminos cada vez más profundos hasta penetrar, en forma cada vez más honda, en el pensamiento de la actualidad europea para el momento, estructurando de manera progresiva una visión de la historia de la filosofía occidental.

Los aportes de Miró Quesada al pensamiento filosófico latinoamericano, con sus afanes dirigidos hacia la gran meta del advenimiento de un filosofar auténtico, comenzaron a dar frutos al lograr la constitución de un proyecto que superara y trascendiera la mera comprensión, para crear una conciencia propia del Ser de estos lugares multiétnicos y de pluriculturalidad. Nadie imaginaria en el siglo XIX, que pocos años después en América Latina pudiera surgir un verdadero movimiento filosófico formados por pensadores latinoamericanos auténticos, terminando así la época idolatría a mentes occidentales para entrar en la época de la autoconciencia latinoamericana.

Miró-Quesada, tiene en Despertar y Proyecto de Filosofar Latinoamericano, su obra cumbre. Junto con Augusto Salazar Bondy, inicia la época en la cual la filosofía de esta parte del mundo logra su propio estilo; ya, no es el calco del pensar de filósofos de otras latitudes. A partir de estos autores, emergió una corriente de expresión filosófica producto de la interpretación de nuestros propios acontecimientos.

Es decir, los intelectuales de la Región, en adelante comenzarían a pensar por sí mismos los grandes temas de la filosofía occidental; llegando a desarrollar la creatividad a través de la total asunción del original pensamiento del filósofo latinoamericano. Esto se debe interpretar como el inicio de un largo proceso de asimilación para llegar a comprender hasta los últimos fundamentos, el pensamiento filosófico en su expresión histórica; y, con ello, lograr descubrir los propios caminos por donde América Latina debe transitar.

Por otra parte, es un reto para la generación de pensadores latinoamericano de hoy, mantener tal como lo proyectó Miró-Quesada, el pensamiento filosófico de esta parte del mundo contextualizado a nuestras realidades, sin olvidar la universalidad del Ser Humano; asimismo, sustentarse en la glocalización como postura de preservación de nuestra acervo identitario. Por lo que expresa Dussel: "América Latina posee una 
razón o racionalidad alternativa a la occidental, pero que sepamos, el único proyecto en parte realizado de una nueva teoría de la razón en América Latina es el de Francisco Miró Quesada Cantuarias" (Sobrevilla, 2007, p, 97)

De esta forma de pensamiento, surgen filósofos de la talla de Vasconcelos y del mismo Dusell, con sus prédicas de no ignorar a la ciencia cuando se estudie la realidad sensible; así como, de la envergadura de León Rozitchner, que invita a reflexionar sobre la relación entre política, economía y teología, en el ámbito de las "Cuestiones cristianas". Con relación a esto Sucksdorf expone:

Teniendo en cuenta estas consideraciones, las líneas que siguen pretender apuntar algunos elementos de la crítica de Rozitchner a la religión que permitan relevar su lugar disruptivo y desestabilizador de aquellos discursos, tan extendidos en América Latina, en que marxismo y religión se reconcilian, junto con sus aportes a la crítica marxista de la religión" (Sucksdorf, 2018, p. 84)

\section{DISCUSIÓN}

El pensamiento de Domingo Faustino Sarmiento como político y educador, conlleva a señalar que su principal preocupación fue la educación. Propuso organizar la República democrática, independiente, con libertad y progreso; asimismo, que había que tener un pueblo con todas las capacidades desarrolladas brindándoles básicamente una educación excelente, y así lograr el bienestar común de los ciudadanos. Por consiguiente, para Sarmiento, las escuelas constituiría la fundamentación de todo buen gobierno republicano.

Igualmente, pensaba que era prioridad que toda la Argentina fuese una gran escuela, para el desarrollo pleno de todos los argentinos. Pues, solo con ciudadanos bien educados se podría sostener una verdadera democracia, plena de derechos y deberes en todos los órdenes sociales y civiles para los connacionales. Esto haría la felicidad de la República.

En lo que respecta a Francisco Miro-Quesada, este se inclinó por una filosofía propia latinoamericana libre de ataduras; destacándose universalmente por su propuesta; $y$, también, en lo que concierne a la lógica formal jurídica. Asimismo, recibió elogios de especialistas en la materia, por sus libros de la historia contemporánea del Perú, en 
la cual expone un gran planteamiento analítico sobre ética, filosofía del conocimiento y sobre la relación de la ética y la política.

Ahora bien, el trabajo más trascendental de este intelectual radica en que analizó con acierto los movimientos descolonizadores latinoamericano de su época estudiando con profundidad la filosofía de la liberación y de la postmodernidad; exaltando con ello las variadas producciones de autenticidad filosófica de pensadores coetáneos. Sus esfuerzos se concentraron en la búsqueda de una identidad sub-continental, siendo el primer abanderado del propósito para lograr un nuevo paradigma de la conciencia filosófica en América Latina.

Es importante destacar que Sarmiento y Miro-Quesada, a pesar que vivieron en épocas diferentes en el tiempo y en las circunstancias, supieron interpretar adecuadamente los escenarios que les correspondió vivir y compartir con destacados intelectuales, gobernantes, funcionaros del Estado y ciudadanos en general. Sarmiento asumió la función pública desde el Estado democrático funcionalista y el servicio gubernamental como un derecho del ciudadano, colocando a la educación como una garantía para el desarrollo.

Mientras que Miro-Quesada le otorgó a la ética y a las ideología una gran importancia en el desenvolvimiento público de los ciudadanos; ganado por el humanismo se inclinó hacia el comunismo hasta que lo abonado luego de largos años de militancia, por oponerse a esta forma de neo-colonización. Su actitud de filósofo le hizo asumir una política como ciencia, de allí la filosofía del fracaso que le atribuyó a esta ideología.

La educación ocupó la atención de Sarmiento en cuanto a la cobertura matricula, la formación del capital humano y el desarrollo social inclusivo de la misma. Asimismo, le dio importancia a los conocimientos, competencias y valores de los individuos; necesarios para alcanzar una sociedad de hombres libres e iguales.

Finalmente, Miro-Quesada también demostró su inclinación por un sistema educativo sólido y que respondiera a los retos de futuro. Como Ministro de Educación, hizo aportes a la filosofía, en el área de las matemáticas y al formalismo ético; además de 
estudiar la naturaleza el hombre. También, se preocupó por el desarrollo de una educación tecnológica, con una visión de futuro hacía las herramientas informáticas.

\section{CONCLUSIÓN}

Al abordar la influencia de Domingo Faustino Sarmiento y Francisco Miró-Quesada Cantuarias, como pensadores vanguardistas en términos políticos y filosóficos; el corolario se centra en afirmar que ambos personajes tuvieron una influencia decisiva en Latinoamérica entre los siglos XIX y XX, acreditándose cada uno como referencia en los ámbitos educativos, políticos y filosóficos que aun hoy en día se mantienen vigentes.

La formación intelectual de ambos fue distinta, mientras que Sarmiento fue autodidacta; Miro-Quesada fue profesor universitario. Sin embargo, fue en el quehacer diario donde plasmaron, producto del pleno ejercicio gubernamental y ciudadano, su legado para las generaciones que los sobrevivieron, el primero en educación básica y el segundo en educación superior y filosofía. En este sentido se puede afirmar que se aprecia una complementariedad intelectual en estos científicos.

Por otra parte, en lo que concierne a lo ideológico, se puede deducir que existió un antagonismo en este orden. Mientras Sarmiento se inclinó por la derecha; MiroQuesada participó como militante de la izquierda, aun cuando se alejó de la misma, catalogándola como la filosofía del fracaso.

Para cerrar, también es importante destacar, que políticamente coincidieron ambos intelectuales, en las proposiciones de inclusión social y humanismo como ideas fundamentadoras de la gestión pública. De allí que se pueda entender que tanto en Sarmiento como en Miro-Quesada, el bien común siempre estuvo por encima de las posturas ideológicas. 


\section{REFERENCIAS CONSULTADAS}

Arias, M. (2001) La Escuela Pública como Productora de Identidades. Contextualización y Análisis del Proyecto de Sarmiento en el Bicentenario de su Nacimiento. Revista Escuela de Historia, Volumen. 10, Número 2. Disponible en: https://n9.cl/m9leq

Cumana, Y. (2008) José Ingenieros: su Visión de la Educación Necesaria para América Latina. Revista Educere, volumen. 12, número. 43, octubre-diciembre, 2008, pp. 831-838 Universidad de los Andes Mérida, Venezuela Disponible en: https://n9.cl/dgpw

Miró-Quesada, F. (1974) Despertar y Proyecto de Filosofar latinoamericano. Ediciones Fondo de Cultura Económica. México

Ocampo, J. (2000). Domingo Faustino Sarmiento. El Presidente de Argentina "Maestro de América". Revista Historia de la Educación Latinoamericana, №. 2, pp. 11-36. Universidad Pedagógica y Tecnológica de Colombia.

Peña, S. (2008) Mariátegui, la Revista Amauta y el Psicoanálisis. Revista de NeuroPsiquiatría, volumen. 71, número. 1-4, pp. 34-42 Universidad Peruana Cayetano Heredia Lima, Perú Disponible en: http://www.redalyc.org/articulo.oa?id=372036927007

Quiroz, R. (2007). A Propósito deFfilosofía peruana. Una Reseña sobre Ser humano, naturaleza e historia. Anales del Seminario de Historia de la Filosofía, volumen 24. pp. 255-260 Universidad Complutense de Madrid, España. Disponible en: http://www.redalyc.org/articulo.oa?id=361133108009

Sarmiento, D. (1995) Conflictos y Armonías de las Razas en América.. Editorial Ediciones Akal, S.A... México.

Sobrevilla, D. (2007) Una Historia de la Filosofía Latinoamericana. Revista de Filosofía Volumen 63 pp. 87-97. Universidad de Lima. Disponible en: https://scielo.conicyt.cl/pdf/rfilosof/v63/art06.pdf

Sucksdorf, C. (2018) Aportes del Pensamiento Crítico Latinoamericano № 5: León Rozitchner: un marxismo con cuerpo propio. Instituto de Estudios de América Latina y el Caribe. Facultad de Ciencias Sociales, Universidad de Buenos Aires: Marcelo T. de Alvear. Disponible en: https://n9.cl/yqep

UNESCO (2017) Informe de Seguimiento de la Educación en el Mundo. Primera Edición' Organización de las Naciones Unidas (ONU). Disponible en: https://n9.cl/62s47 
CIENCIAMATRIA

Revista Interdisciplinaria de Humanidades, Educación, Ciencia y Tecnología

Año VI. Vol. VI. N${ }^{\circ}$. Edición Especial. 2020

Hecho el depósito de ley: pp201602FA4721

ISSN-L: 2542-3029; ISSN: 2610-802X

Universidad Nacional Experimental Francisco de Miranda (UNEFM). Santa Ana de Coro. Venezuela

Leonardo Díaz-Petruz; Luz Estela Díaz-Cid; Luz Marina-Mejías; Miguel Negrón

Villarán. A. (2019). El principio Ético de no Arbitrariedad: la Teoría Moral Formal de Francisco Miró Quesada. Revista Pensamiento volumen I. 75, número. 286, pp. 1339-1360. Universidad del Pacífico, Lima, Perú. https://n9.cl/vmh5

(C2020 por el autor. Este artículo es de acceso abierto y distribuido según los términos y condiciones de la licencia Creative Commons Atribución-NoComercial-Compartirlgual 4.0 Internacional (CC BY-NC-SA 4.0)

(https://creativecommons.org/licenses/by-nc-sa/4.0/). 